\title{
How nano can fight the virus
}

A s well as being a triumph of speed and ingenuity, the approved vaccines against the SARS-CoV-2 developed by the German biotechnology company BioNTech, working with Pfizer, and by US-based Moderna mark "the coming of age of nanomedicine", in the words of materials physicist Richard Jones. Both vaccines incorporate strands of mRNA encoding the 'spike' docking protein of the virus, so that this antigen is produced in the body of a vaccinated person, thereby raising an immune response. To introduce the mRNA into the host cells, it is packaged in engineered nanoparticles. These are tiny compartments (liposomes) made primarily from phospholipids and given a 'hairy coat' of the polymer polyethyelene oxide, which may help to ward off the body's defences. The nanoparticles will self-assemble in the presence of the mRNA and encapsulate it. The details of the formulations are proprietary, but there's every reason to think that these particles can act as a general (and now, a proven safe) chassis for other RNA vaccines, potentially speeding up future vaccine development still further.

This is the most dramatic but by no means the only potential way in which nanoparticles can help combat the dreadful pandemic. One of the advantages of using nanoparticles in antiviral therapeutics is that they may be of much the same size as the virus itself and can therefore get up close to attack it. One strategy being considered is to use nanoparticles covered with the human membrane protein called ACE2 - to which the coronavirus binds in the airways and lungs to enter cells - to mop up the virus in these parts of the airspace. The trapped virus would then be cleared by being caught by the mucus layer that sweeps foreign debris into the digestive tract. Antiviral agents like this might potentially be delivered as an inhaled aerosol, thereby arriving precisely at the locations where they are needed to attack a virus that targets the respiratory system. This requires a better understanding of the transport mechanisms, for which some ex vivo experiments with mouse lung tissue have already delivered preliminary results ${ }^{1}$. Another strategy, already pursued for cancer therapeutics ${ }^{2}$ as well as for influenza antivirals, is to make inorganic nanoparticles that will bind to the virus and then absorb tissue-penetrating radiation (such as near-infrared) to heat up and destroy it. Indeed, the breadth of nanoparticle-based antiviral strategies is vast ${ }^{3}$.

Protein- and polymer-based nanoparticles, either injected or inhaled, are also being studied for controlling the over-reaction of the immune system that leads to acute and sometimes lethal cases of COVID-19 $9^{4,5}$. The aim here is to find nanoparticles that will divert the attention of the white blood cells called neutrophils that can otherwise respond to the virus by causing life-threatening inflammation in the lungs (acute respiratory distress syndrome).

The potential of nanoparticles for COVID-19 vaccines is now broadened by the finding of Huang et al. ${ }^{6}$ that they can substantially boost the immune response to the receptor-binding domain (RBD) of the SARS-CoV-2 spike protein. In experiments on mice, the researchers found that liposomes containing phospholipids functionalized with cobalt porphyrin groups can be combined with RBD proteins to make nanoparticles that may enter



Philip Ball

the antigen-presenting cells that elicit antibodies against the virus, and enhance their response relative to lone molecules of the soluble RBD protein domain. This results in higher antibody levels, better blocking of viral binding to ACE2, and better inhibition of viral replication. The cobalt-containing nanoparticles don't seem to show signs of triggering adverse reactions or toxicity in the mice, and could reduce the dose needed for vaccines based on the RBD of the spike protein.

It's early days yet for this or many of the other nanoparticle approaches. But the ability to control and engineer interactions and properties at the nanoscale has already proved its worth in the pandemic, and we can feel confident there is more to come.

Published online: 27 January 2021 https://doi.org/10.1038/s41563-020-00916-X

References

1. Minahan, D., Donzanti, M. J., Fromen, C. A. \& Gleghorn, J. P. Am. J. Respir. Crit. Care Med. 201, A5690 (2020).

2. Vines, J. B., Yoon, J.-H., Ryu, N.-E., Lim, D.-J. \& Park, H. Front. Chem. 7, 167 (2019).

3. Wieczorek, K., Szitkowska, B. \& Kierzek, E. Pathogens 9 , 1020 (2020).

4. Fromen, C. A. et al. ACS Nano 11, 10797-10807 (2017).

5. Myserson, J. W. et al. Preprint at https://doi.org/10.1101/ 2020.04.15.037564 (2020).

6. Huang, W.-C. et al. Adv. Mat. 32, 2005637 (2020). 